\title{
Sex differences in adolescents' glycaemic and insulinaemic responses to high and low glycaemic index breakfasts: a randomised control trial
}

\author{
Simon B. Cooper*, Karah J. Dring, John G. Morris, Ben E. W. Cousins, Maria L. Nute and Mary E. Nevill \\ Exercise and Health Research Group, Department of Sports Science, Sport Health and Performance Enhancement (SHAPE) \\ Research Centre, Nottingham Trent University, Nottingham NG11 8NS, UK
}

(Submitted 3 October 2016 - Final revision received 9 January 2017 - Accepted 6 February 2017-First published online 13 March 2017)

\begin{abstract}
During puberty young people undergo significant hormonal changes which affect metabolism and, subsequently, health. Evidence suggests there is a period of transient pubertal insulin resistance, with this effect greater in girls than boys. However, the response to everyday high and low glycaemic index (GI) meals remains unknown. Following ethical approval, forty adolescents consumed a high GI or low GI breakfast, in a randomised cross-over design. Capillary blood samples were taken during a 2-h postprandial period, examining the glycaemic and insulinaemic responses. Maturity offset and homoeostatic model assessment (HOMA) were also calculated. The glycaemic response to the breakfasts was similar between boys and girls, as shown by similar peak blood glucose concentrations and incremental AUC (IAUC) following both high and low GI breakfasts (all $P>0.05$ ). Girls exhibited a higher peak plasma insulin concentration 30 min post-breakfast following both high GI $(P=0 \cdot 043, g=0 \cdot 69)$ and low GI $(P=0 \cdot 010, g=0 \cdot 84)$ breakfasts, as well as a greater IAUC following high GI $(P=0 \cdot 041, g=0 \cdot 66)$ and low GI $(P=0 \cdot 041, g=0 \cdot 66)$ breakfasts. HOMA was positively correlated with the insulinaemic responses $($ all $P<0 \cdot 0005)$ and maturity offset $(P=0.037)$. The findings of the present study suggest that pubertal insulin resistance affects the postprandial insulinaemic responses to both high and low GI meals. Specifically, girls exhibit a greater insulinaemic response than boys to both meals, despite similar glycaemic responses. This study is the first to report the glycaemic and insulinaemic responses to everyday meals in boys and girls, supporting the recommendation for young people to base their diet on low GI carbohydrates.
\end{abstract}

Key words: Insulin resistance: Puberty: Breakfast glycaemic index: Sex differences

During puberty and maturation, young people undergo a number of hormonal changes, such as augmented testosterone and free testosterone in pubertal boys and elevated estradiol concentrations in pubertal girls ${ }^{(1)}$. The hormonal changes that occur during puberty have significant effects upon metabolism, and subsequently health, in both sexes ${ }^{(1,2)}$. There is a significant body of evidence to suggest that as a result of these changes, during adolescence there is a transient period of insulin resistance, which begins at the onset of puberty ${ }^{(2-7)}$. Insulin resistance and reduced glucose tolerance are typically implicated in the aetiology of type 2 diabetes, which is becoming more common in adolescents ${ }^{(8)}$. Furthermore, type 2 diabetes in young people is associated with an increased risk of a number of co-morbidities such as $\mathrm{CVD}^{(9)}$. Due to the potential concern for metabolic health across the lifespan, it is important to understand the effect that hormonal changes and the transient period of insulin resistance has upon postprandial metabolism in adolescents.

It has been suggested that adolescents exhibit a higher degree of insulin resistance compared with pre-pubertal children and adults ${ }^{(3)}$. Furthermore, in a cross-sectional comparison of 10-14-year-old boys and girls, it was found that insulin resistance increases at the onset of puberty, reaches a peak at Tanner stage 3 and then returns to baseline levels post puberty $^{(2)}$. The findings of longitudinal studies, tracking young people as they go through puberty, also supports the notion of a period of transient insulin resistance during adolescence ${ }^{(4-7)}$. For example, Goran \& Gower ${ }^{(5)}$ documented a 32\% higher insulin resistance at Tanner stage 3 compared with Tanner stage 1 and Hannon et al. ${ }^{(6)}$ reported a $50 \%$ reduction in insulin sensitivity during puberty.

However, a limitation of the research in the area to date is the choice of technique used to assess pubertal insulin resistance. A number of techniques have been employed, including euglycaemic/hyperglycaemic clamp techniques ${ }^{(2,3,6)}$, intravenous glucose tolerance tests ${ }^{(4,5)}$ and the homoeostatic model assessment (HOMA) ${ }^{(7,10)}$. A limitation of each of these techniques is that they lack ecological validity and to date, the glycaemic and insulinaemic responses of boys and girls to everyday mixed meals (those containing a variety of

Abbreviations: GI; glycaemic index; HOMA; homoeostatic model assessment; IAUC; incremental AUC.

* Corresponding author: Dr S. B. Cooper, fax +44 115848 6636, email Simon.Cooper@ntu.ac.uk 
macronutrients) is yet to be reported, but is of clear importance to health in young people.

Typically, studies are in agreement that the degree of insulin resistance experienced during puberty is greater in girls compared with boys ${ }^{(2,4,10)}$. For example, glucose uptake in response to a euglycaemic clamp was found to be $20 \%$ lower in girls than boys aged 10-14 years, indicative of greater insulin resistance, an effect which was evident across Tanner stages ${ }^{(2)}$. The data of Metcalf et $a l^{(10)}$ also demonstrate that insulin resistance is approximately $30 \%$ greater in girls compared with boys, an effect which is evident in both physically active and inactive adolescents. The greater insulin resistance reported in female adolescents is also consistent with findings in adults diagnosed with type 2 diabetes, with more women failing to achieve the glycosylated $\mathrm{Hb}$ target of $7 \%$ (a marker of long-term glycaemic control), in comparison with their male counterparts ${ }^{(11)}$

Recent evidence continues to suggest that young people typically eat a carbohydrate-rich diet and that the carbohydrates consumed are typically moderate-high glycaemic index $(\mathrm{GI})^{(12)}$. Furthermore, high GI diets are implicated in the aetiology of conditions such as CVD and type 2 diabetes (as well as key risk factors for these conditions), due to the elevated glycaemic and insulinaemic responses seen ${ }^{(13)}$. Given the period of transient insulin resistance during puberty, it is therefore imperative to understand how this affects the glycaemic and insulinaemic responses, of young people, to meals which differ in GI. However, this is yet to be reported in the literature, along with whether the sex differences exist in response to meals differing in GI.

A number of mechanisms have been proposed as causing the transient insulin resistance experienced during puberty. Evidence suggests that during puberty there is less glucose uptake per unit of insulin ${ }^{(14)}$, resulting in lower glucose oxidation and an increase in fatty acid oxidation ${ }^{(15)}$. This drop in insulin resistance seems to be compensated for by an increase in insulin secretion ${ }^{(3,6)}$. A number of studies relate the increase in insulin resistance to an increase in adiposity ${ }^{(7)}$, which may also help to explain the greater insulin resistance in girls compared with boys, given that girls typically display a higher adiposity during this time ${ }^{(16)}$. However, in the study of Moran et al. ${ }^{(2)}$ girls exhibited a greater insulin resistance than boys even when triceps and subscapular thickness were controlled for, thus suggesting that adiposity does not completely explain the greater insulin resistance in girls. This finding is echoed in a subsequent study using dual energy X-ray absorptiometry to assess body composition, also concluding that adiposity does not explain the transient insulin resistance during puberty ${ }^{(6)}$. Another mechanism believed to affect the transient insulin resistance during puberty is an elevated concentration of growth hormone and insulin-like growth factor 1 , with these hormones reported to have a negative effect on insulin sensitivity ${ }^{(7,14,17)}$.

Overall, whilst there seems consensus in the literature regarding the presence of a period of insulin resistance during puberty, a limitation of the literature to date is that the methods used to assess insulin resistance lack ecological validity and the responses to everyday meals remain unknown. Furthermore, whether sex differences exist in response to high and low GI meals in adolescents has not been explored. Therefore, the aim of the present study is to examine the sex differences in the glycaemic and insulinaemic responses to standard high and low GI meals in adolescents.

\section{Methods}

\section{Study design}

This study was conducted according to the guidelines laid down in the Declaration of Helsinki and all procedures involving human subjects/patients were approved by the institution's ethical advisory committee (approval no. R09-P118). Participants were recruited from two local schools and school-level consent was obtained from head teachers. In addition, written parental informed consent, participant assent and health screen questionnaire forms were completed for each participant. The trial was registered with the International Standard Randomised Controlled Trials Registry (identification no. ISRCTN17903058, available at: www.isrctn.com/ISRCTN17903058).

The study employed an order-balanced, randomised, crossover design with all participants completing a familiarisation, which preceded the first of three experimental trials by $7 \mathrm{~d}$. All trials took place at the school the children attended and the experimental trials consisted of a high GI, low GI and breakfast omission trial, each separated by $7 \mathrm{~d}$. Therefore, participants acted as their own controls. The findings regarding the effects of breakfast GI on cognitive function have been reported elsewhere ${ }^{(18)}$. The present paper examines the glycaemic and insulinaemic response to the high GI and low GI breakfasts and the difference in responses between sexes, data which were not previously reported.

Following an overnight fast participants arrived at school at the normal time and rested in a seated position for $10 \mathrm{~min}$, following which a fasted capillary blood sample was taken. Participants were then given $15 \mathrm{~min}$ to consume the breakfast, following which further capillary blood samples were taken at 15, 30, 60 and $120 \mathrm{~min}$ from the start of breakfast. A $120 \mathrm{~min}$ postprandial monitoring period was chosen based upon previous work suggesting this is a sufficient period of time to examine the different glycaemic response between meals ${ }^{(18)}$.

\section{Participants}

In total, fifty-two participants aged 12-14 years old were recruited to participate in the study. Eligibility criteria were to be aged 12-14 years old and any participants with health issues or food allergies and intolerances that could affect their participation were excluded from the study. In all, twelve participants did not complete the study (ten were absent from school for one of the experimental trials and two did not follow the dietary requirements). Therefore, forty participants completed the study and were included in the data analysis.

During the familiarisation session, participant anthropometrics were measured. Height was measured using a Leicester Height Measure (Seca), accurate to $0 \cdot 1 \mathrm{~cm}$ and body mass was measured using a Seca 770 digital scale (Seca), accurate to $0 \cdot 1 \mathrm{~kg}$, to allow the calculation of BMI. Sitting height was also measured to allow calculation of leg length and subsequently 
maturity offset, using the method of Moore et $a l^{(19)}$. Waist circumference was also measured, to the nearest $0.1 \mathrm{~cm}$, at the narrowest part of the torso between the xiphoid process of the sternum and the iliac crest. Physiological data for the participants, along with a comparison between males and females, are displayed in Table 1.

\section{Pre-trial control}

The evening before each experimental trial, participants were asked to consume the same meal and follow an overnight fast from 22.00 hours. Water was allowed ad libitum at all times. Participants were also asked to avoid any unusually vigorous physical activity for the $24 \mathrm{~h}$ preceding each experimental trial. The evening before each experimental trial the research team made a telephone call to participants to remind them of this information and verbal confirmation of compliance was sought upon arrival at school.

\section{Breakfast}

The high and low GI breakfasts each contained $1.5 \mathrm{~g} / \mathrm{kg}$ body mass carbohydrate and were matched for energy and macronutrient content. The GI of the meals was calculated using previously described methods ${ }^{(20,21)}$ and GI values for each food were taken from internationally available tables ${ }^{(22)}$. Participants were given $15 \mathrm{~min}$ to consume the breakfasts. Furthermore, $150 \mathrm{ml}$ water was provided with the high GI breakfast to ensure matched water intake between trials and $150 \mathrm{ml}$ water was provided at $60 \mathrm{~min}$ on both trials to maintain euhydration. Table 2 displays the breakfast composition for a $50 \mathrm{~kg}$ participant.

\section{Capillary blood samples}

Capillary blood samples were preferred to venous samples in the present study because they are more sensitive to glycaemic responses and are more acceptable within the ethical constraints of working with young people ${ }^{(23-26)}$. A fasted capillary blood sample was taken at baseline, followed by subsequent samples 15, 30, 60 and 120 min following the start of breakfast.

Participants' hands were warmed via submersion in warm water to increase capillary blood flow. A Unistik single use lancet (Unistik Extra, 21G gauge, $2.0 \mathrm{~mm}$ depth; Owen Mumford Ltd) was used and the blood collected into two $300 \mu \mathrm{l}$ EDTA coated microvettes (Sarstedt Ltd). Two $25 \mu$ l whole blood samples were removed using plain pre-calibrated glass pipettes (Hawksley Ltd), immediately deproteinised in $250 \mu$ of $2.5 \%$ ice cooled perchloric acid in $1.5 \mathrm{ml}$ plastic vials and centrifuged at $7000 \mathrm{rpm}$ for $4 \mathrm{~min}$ (Eppendorph 5415C). The remaining whole blood was also centrifuged at $7000 \mathrm{rpm}$ for $4 \mathrm{~min}$ (Eppendorph 5415C) and the plasma removed and placed into $500 \mu \mathrm{l}$ plastic vials. All samples were frozen at $-20^{\circ} \mathrm{C}$ until analysis.

All bloods samples were analysed in duplicate for blood glucose and plasma insulin concentration. Blood glucose concentrations were determined using a commercially available kit (GOD-PAP method, GL 2610; Randox) and were analysed spectrophotometrically (Cecil CE393 digital grating spectrophotometer; Cecil Instruments Ltd), with an inter-assay CV of

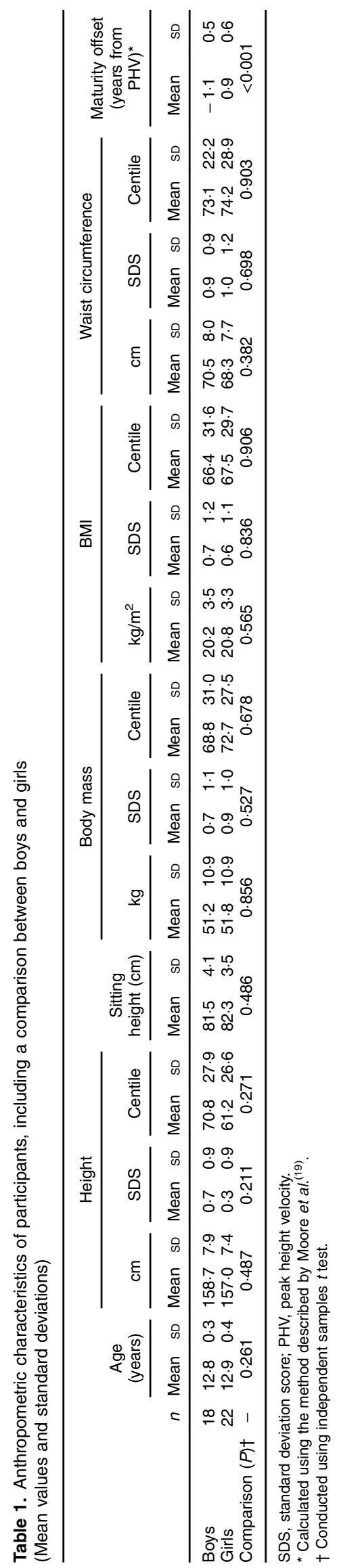


Table 2. Composition of high glycaemic index (HGl) and low glycaemic index (LGI) breakfasts for a $50 \mathrm{~kg}$ participant

\begin{tabular}{|c|c|c|}
\hline Breakfasts & $\mathrm{HGI}$ & LGI \\
\hline \multicolumn{3}{|l|}{ Food items (g) } \\
\hline Cornflakes ${ }^{*}$ & 55 & \\
\hline White bread $\dagger$ & 42 & \\
\hline Margarine $\ddagger$ & 6 & \\
\hline $1 \%$ fat milk§ & 216 & 217 \\
\hline Mueslill & & 75 \\
\hline Appleף & & 150 \\
\hline \multicolumn{3}{|l|}{ Macronutrients } \\
\hline Energy (kJ) & 1766 & 1757 \\
\hline Energy (kcal) & 422 & 420 \\
\hline Cholesterol (g) & 75.0 & $75 \cdot 0$ \\
\hline Fat $(\mathrm{g})$ & $7 \cdot 2$ & 6.4 \\
\hline Protein $(\mathrm{g})$ & $14 \cdot 3$ & $15 \cdot 5$ \\
\hline Glycaemic index** & 72 & 48 \\
\hline Glycaemic loadt† & 54 & 36 \\
\hline
\end{tabular}

* Cornflakes (Kelloggs Ltd).

† Lightly toasted white bread (Kingsmill soft white thick slice).

$\ddagger$ Margarine (Flora Original).

$\S 1 \%$ fat milk (Sainsbury's Ltd).

II Muesli (Alpen no added sugar; Weetabix Ltd).

II Apple (Braeburn apple).

** Calculated by the method previously described ${ }^{(20,21)}$ with glycaemic index values taken from international tables ${ }^{(22)}$

t† Calculated by the method previously described ${ }^{(22)}$

$2.3 \%$ and an intra-assay CV of $1.2 \%$. Plasma insulin concentrations were determined using an ELISA (Mercodia Ltd), with an inter-assay CV of $3.2 \%$ and an intra-assay CV of $1.9 \%$. Incremental AUC (IAUC) for blood glucose and plasma insulin was calculated using previously described methods ${ }^{(20)}$. Insulin resistance was assessed using the HOMA method ${ }^{(27)}$. The HOMA calculation requires fasting blood glucose and plasma insulin concentrations and the data presented here are the mean HOMA values upon arrival for the high and low GI trials each child.

\section{Statistical analysis}

A power calculation was performed to determine the appropriate sample size, based on the previously reported effects in young people ${ }^{(17)}$. Based on an estimated effect size of 0.8 (and two-tailed significance), the power analysis yielded a required total sample size of forty (twenty girls and twenty boys).

All data analyses were performed in SPSS (version 23; SPSS Inc.). The primary outcome variables of interest were blood glucose and plasma insulin concentrations, whilst the secondary outcome variable was HOMA. Blood glucose and plasma insulin concentration data were analysed using a three-way (breakfast $\times$ time $\times$ sex) mixed methods ANOVA, with repeated measures for breakfast and time. IAUC data were analysed using a two-way (breakfast $\times$ sex) mixed methods ANOVA. All pairwise comparisons between boys and girls were performed using independent samples $t$ tests and correlations were performed using Pearson's correlation. Effect sizes are reported as Hedges' $g$. Statistical significance was accepted as $P<0.05$ and all data are presented as mean values and standard deviations, unless stated otherwise.

\section{Results}

Blood glucose

Blood glucose concentration. Fasted blood glucose concentration was not different between the high and low GI trials $(P=0 \cdot 415)$. Blood glucose concentration was greater on the high GI trial when compared with the low GI trial (main effect of trial, $\left.F_{1,37}=47.9, P<0.0005\right)$ and increased following both breakfasts (main effect of time, $F_{1,37}=47.9, P<0.0005$ ) but there was no overall difference in blood glucose concentration between the sexes (main effect of sex, $P=0.353$ ). There was a significant breakfast $\times$ time interaction $\left(F_{4,148}=6.759, P<0.0005\right)$ whereby blood glucose concentration reached a higher peak and remained higher following the high GI breakfast. However, no interactions were seen between breakfast and sex $(P=0.084)$ or time and sex $(P=0.520)$. There was a significant three-way breakfast $\times$ time $\times$ sex interaction $\left(F_{4,148}=3 \cdot 0, P=0 \cdot 019\right)$ whereby boys exhibited a greater difference in response between the high and low GI trials when compared with girls (Fig. 1). There was no difference in the peak blood glucose concentration (at $30 \mathrm{~min}$ ) between the boys and girls following the high GI $(P=0 \cdot 131)$ or low GI breakfasts $(P=0.682)$ breakfasts.

Incremental AUC. Blood glucose IAUC was greater following the high GI breakfast when compared with the low GI breakfast (high GI: $121 \mathrm{mmol} / 1 \times 120 \mathrm{~min}$, low GI: $80.3 \mathrm{mmol} / 1 \times 120 \mathrm{~min}$, main effect of trial, $\left.F_{1,37}=40 \cdot 2, P<0 \cdot 0005\right)$. Blood glucose IAUC was not different between sexes (main effect of sex, $P=0 \cdot 361$ ) nor was there a sex difference in blood glucose IAUC between the high and low GI trials (breakfast $\times$ sex interaction, $P=0 \cdot 108)$. Furthermore, blood glucose IAUC was not different between the sexes following the high GI $(P=0 \cdot 187)$ or low GI $(P=0 \cdot 895)$ breakfasts.

\section{Plasma insulin}

Plasma insulin concentration. Fasted plasma insulin concentration was not different between the high and low GI trials $(P=0 \cdot 190)$. Overall, there was no difference in plasma insulin concentration between the high GI and low GI trials (main effect of breakfast, $P=0.094$ ), but plasma insulin concentration was elevated following both breakfasts (main effect of time, $\left.F_{4,152}=67.8, P<0.0005\right)$. In addition, overall plasma insulin concentrations were higher in girls compared with boys (main effect of sex, $\left.F_{1,38}=5 \cdot 5, P=0 \cdot 025\right)$. The pattern in change of plasma insulin concentration was not different between the high and low GI trials (breakfast $\times$ time interaction, $P=0.739$ ), nor was there a breakfast $\times$ sex interaction $(P=0.755)$. Plasma insulin concentrations exhibited a significant time $\times$ sex interaction $\left(F_{4,38}=4 \cdot 2, P=0.003\right)$, whereby girls exhibited a greater plasma insulin concentration than boys at all time points following breakfast (Fig. 2). However, this effect was not different between the high and low GI trials (breakfast $\times$ time $\times$ sex interaction, $P=0 \cdot 686$ ). Plasma insulin concentration reached a higher peak (at $30 \mathrm{~min}$ ) in girls compared with boys following both the high GI (girls: 839 (sD 488) pmol/l, boys: 551 (sD 353) $\left.\mathrm{pmol} / \mathrm{l} ; t_{1,38}=2 \cdot 1, \quad P=0.043, g=0.69\right)$ and low GI 


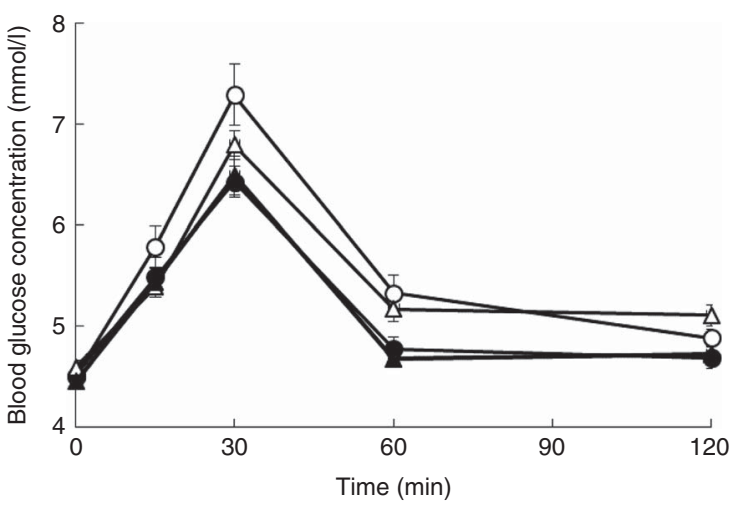

Fig. 1. Blood glucose concentration following the high glycaemic index ( $\mathrm{HGl})$ and low glycaemic index (LGl) breakfasts in boys (B) and girls (G). Values are means with their standard errors. Breakfast $\times$ time $\times$ sex interaction, $P=0.019$. $\multimap$-, HGI-B; ——, LGI-B; $\neg-$, HGI-G; —, LGI-G.

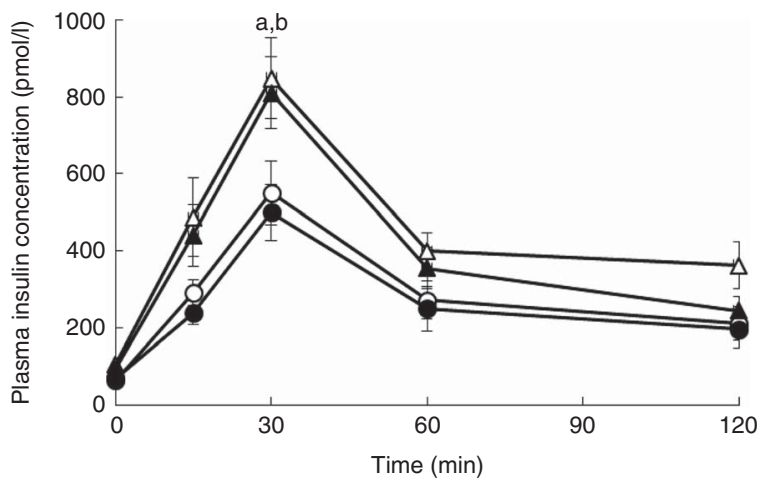

Fig. 2. Plasma insulin concentration following the high glycaemic index ( $\mathrm{HGl}$ and low glycaemic index (LGI) breakfasts in boys (B) and girls (G). Values are means with their standard errors. Time $\times$ sex interaction, $P=0.003$; a HGI-G $>$ HGI-B, $P=0.043$; ${ }^{b}$ LGI-G $>$ LGI-B, $P=0.010$. - - - HGI-B; $\multimap-$ LGI-B; $\neg-$, HGI-G; $\multimap$, LGI-G.

(girls: 831 (sD 438) pmol/l, boys: 501 (sD 309) pmol/l; $t_{1,38}=2 \cdot 7$, $P=0 \cdot 010, g=0 \cdot 84)$ breakfasts.

Incremental AUC. Plasma insulin IAUC was greater following the high GI breakfast when compared with the low GI breakfast (high GI: $36 \cdot 2 \mathrm{nmol} / 1 \times 120 \mathrm{~min}$, low GI: $31 \cdot 0 \mathrm{nmol} / 1 \times 120 \mathrm{~min}$, main effect of breakfast, $\left.F_{1,38}=6.593, P=0.014\right)$ and was also greater in girls compared with boys (girls: $41 \cdot 1 \mathrm{nmol} / 1 \times 120 \mathrm{~min}$, boys: $26 \cdot 1 \mathrm{nmol} / 1 \times 120 \mathrm{~min}$, main effect of sex, $F_{1,38}=4 \cdot 8$, $P=0.034)$. However, there was no sex difference in plasma insulin IAUC between the high and low GI trials (breakfast $\times$ sex interaction, $P=0.491)$. Girls exhibited a higher plasma insulin IAUC following both the high GI (girls: 44.5 (sD 27.4) nmol/1 $\times$ $120 \mathrm{~min}$, boys: $28 \cdot 0 \quad(\mathrm{sD} 20 \cdot 4) \mathrm{nmol} / 1 \times 120 \mathrm{~min}, \quad t_{1,38}=2 \cdot 1$, $P=0.041, g=0 \cdot 66$; Fig. 3) and low GI (girls: $37 \cdot 8$ (sD 19.8) nmol $/ 1 \times$ $120 \mathrm{~min}$, boys: $24 \cdot 2$ (sD 20.7) $\mathrm{nmol} / 1 \times 120 \mathrm{~min}, \quad t_{1,38}=2 \cdot 1$, $P=0.041, g=0 \cdot 66$; Fig. 3) breakfasts, when compared with boys.

\section{Homoeostatic model assessment}

There was a tendency for HOMA to be higher in females (1.63 (SD 0.86) AU) than boys (1.22 (SD 0.49) AU), but this did not

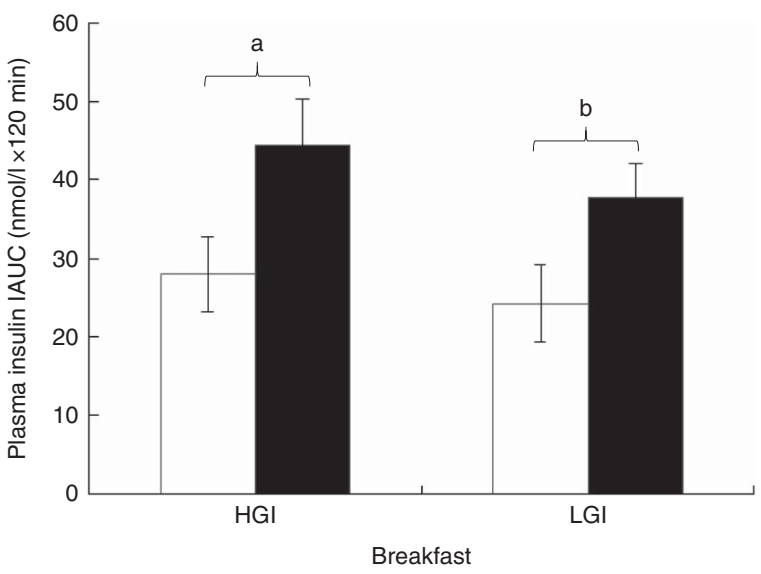

Fig. 3. Plasma insulin incremental AUC (IAUC) following the high (HGI) and low glycaemic index (LGI) breakfasts in boys $(\square)$ and girls $(\square)$. Values are means with their standard errors. ${ }^{a} \mathrm{HGl}$-girls $>\mathrm{HGI}$-boys, $P=0.041 ;{ }^{b}$ LGIgirls $>$ LGI-boys, $P=0.041$.

reach statistical significance $(P=0.082)$. Interestingly, there were strong positive correlations observed between HOMA and plasma insulin IAUC following both the high GI $(r 0.873$, $P<0.0005)$ and low GI $(r 0.674, P<0.0005)$ breakfasts and between HOMA and peak plasma insulin concentration following both the high GI $(r 0.886, P<0.0005)$ and low GI breakfasts $(r 0.605, P<0.0005)$. No significant correlations were observed between HOMA and blood glucose IAUC (high GI: $r$ 0.076, $P=0.644$; low GI: $r$ 0.185, $P=0.259)$ or peak blood glucose concentration (high GI: $r$ 0.013, $P=0.938$; low GI: $r 0 \cdot 018, P=0.912$ ) following either the high or low GI breakfasts. There was also a weak but statistically significant positive correlation observed between HOMA and maturity offset ( $r 0.332, P=0.037)$, with more mature individuals exhibiting a greater HOMA.

\section{Discussion}

The main finding of the present study is that during puberty, there are marked sex differences in the insulinaemic response to standard high and low GI meals, when comparing boys and girls of similar chronological ages. Girls exhibit a greater insulinaemic response than boys, with a 30-40\% higher peak plasma insulin concentration and IAUC following both the high and low GI breakfasts when compared with the boys. However, the glycaemic response was similar between the sexes. The findings of the present study also suggest that maturation affects the degree of insulin resistance experienced, partly accounting for the sex differences observed in the insulinaemic response to the meals in the present study given that the girls were more mature than boys despite their similar chronological ages. The findings of the present study are novel because they demonstrate the glycaemic and insulinaemic responses to everyday meals and also demonstrate that girls exhibit a greater insulinaemic response following both the high and low GI breakfasts when compared with boys. Therefore, the ecologically valid findings of the present study have important implications in young people. 
The findings of the present study suggest that the girls demonstrate a greater insulinaemic response than boys to both the high and low GI breakfasts. Specifically, peak plasma insulin concentration following the high and low GI breakfasts is greater in girls compared with boys (high GI: 34\%; low GI: $40 \%$ ), and also that the plasma insulin IAUC is greater in girls following both the high and low GI breakfasts, when compared with boys (high GI: 37\%; low GI: 36\%). The present study administered matched glucose loads in the form of the high and low GI breakfasts and despite similar glycaemic responses between boys and girls (peak blood glucose concentration and IAUC), the girls demonstrated the greater insulinaemic responses, indicative of a greater degree of insulin resistance. The degree of sex difference in insulin resistance demonstrated in the present study is slightly greater than the $20-30 \%$ reported in the literature to date ${ }^{(2,4,10)}$. However, the findings of the present study are novel because they demonstrate that the sex differences exist in response to everyday mixed meals (in addition to the clamp techniques used in previous studies).

The findings of the present study also suggest that girls exhibit a greater insulinaemic response, compared with boys, when both high and low GI breakfasts are consumed, which has not previously been examined. It is interesting to note that young people in Western societies typically consume a carbohydraterich diet and more specifically, a diet with a high $\mathrm{GI}^{(12)}$. This is of concern because evidence also links a high GI diet with noncommunicable diseases such as CVD and type 2 diabetes (as well as key risk factors for these conditions), due to the elevated glycaemic and insulinaemic responses seen ${ }^{(9,13)}$. The findings of the present study demonstrate a lower glycaemic and insulinaemic response to the low GI compared with high GI breakfast in both boys and girls, therefore suggesting that young people should aim to consume carbohydrates with a lower GI, especially during this period of pubertal insulin resistance.

When comparing boys and girls of similar chronological ages, the findings of the present study suggest that girls exhibit a much greater insulinaemic response to high and low GI breakfasts than boys at age 11-14 years, whilst the responses in adults are similar between sexes ${ }^{(28,29)}$. It is important to compare boys and girls of the same chronological age because this is how they are grouped in everyday life, such as at school. However, when examining the period of transient pubertal insulin resistance it is also important to consider the effect of maturity upon this, with several studies to date demonstrating that the transient pubertal insulin resistance peaks around tanner stage $3^{(2,5)}$. However, not all studies agree with this and other authors suggest that the degree of pubertal insulin resistance does not differ across Tanner stages ${ }^{(4)}$.

In the present study the estimation of years from peak height velocity was made using the leg length calculations of Moore et $a l .{ }^{(19)}$. This method was chosen as a non-invasive and valid measure which is replicable on a large scale. Other possible techniques to assess maturity have several limitations associated with them, such as: skeletal wrist X-rays which carry ethical concerns and are not replicable on a large scale; age of menarche which cannot be used for between sex comparisons; Tanner stages which are a more invasive measure and evidence suggests they should not be used for between sex comparisons $^{(30)}$; and the Khamis-Roche equation which requires biological parent height which may not always be possible and may cause a sensitive issue where young people do not live with their biological parents.

The data using the Moore et al. method $^{(19)}$ unsurprisingly demonstrated that the girls ( 0.9 years post peak height velocity) were more mature than boys ( 1.1 years pre peak height velocity) given their similar chronological ages. Interestingly, there was also a weak but statistically significant correlation between the calculated maturity offset and HOMA. Therefore, the findings of the present study add weight to the argument that the degree of pubertal insulin resistance increases in the more mature individuals, but given the cross-sectional nature of this study we cannot comment specifically on the time-course of these effects. Unfortunately, the present study did not yield sufficiently sensitive data to allow comparisons between boys and girls matched for maturation (because the young people were recruited to the study based on their school year and thus there was insufficient overlap in maturity between the boys and girls). However, this is something that could be examined in future research to further explore the mediating effect of maturation on sex differences in insulin resistance. It may also be of interest to examine the effects of the menstrual cycle on the glycaemic and insulinaemic responses in girls following menarche, given the potential for the menstrual cycle to affect substrate utilisation $^{(31)}$.

There are several suggestions in the literature to date that the pubertal insulin resistance is compensated for by an increase in insulin secretion ${ }^{(3,6)}$. The findings of the present study suggest that this is also the case in response to both high and low GI meals whereby, although girls exhibit a much greater insulinaemic response, the glycaemic responses are similar between boys and girls. It is also important to note that the breakfasts provided in the present study contained $1.5 \mathrm{~g} / \mathrm{kg}$ body mass of carbohydrate and given there were no differences in body mass between the boys and girls, a similar carbohydrate load was provided to both. However, during pubertal development boys typically exhibit an increase in metabolically active tissue whilst adiposity increases in girls, although there were no differences in waist circumference (a marker of central adiposity) between the boys and girls in the present study. Furthermore, several studies to date have concluded that adiposity does not explain the transient insulin resistance during adolescence ${ }^{(2,6)}$. In future research meals which contain a set amount of carbohydrate per unit of lean body mass could be provided to continue to examine the effect of adiposity on pubertal insulin resistance and further improve the experimental control.

\section{Conclusions}

Overall, the findings of the present study suggest that there are marked sex differences in the insulinaemic response to high and low GI meals in adolescents, whereby despite similar glycaemic responses girls exhibit a 30-40\% greater insulinaemic response. These findings are important because the present study is the first to document the responses to everyday mixed meals and also demonstrate that the sex differences are evident following both high and low GI breakfasts. Thus, the present 
study provides further evidence for the current recommendation that adolescents should consume a low GI carbohydrates to optimise their cardio-metabolic health.

\section{Acknowledgements}

S. B. C., J. G. M. and M. E. N. designed the research; S. B. C., M. L. N. and J. G. M. conducted the research; S. B. C., B. E. W. C. and K. J. D. analysed the data and S. B. C., K. J. D., B. E. W. C. and M. E. N. had primary responsibility for writing the paper. All authors read and approved the final manuscript.

The authors declare that there are no conflicts of interest.

\section{References}

1. Roemmich JN \& Ragol AD (1999) Hormonal changes during puberty and their relationship to fat distribution. Am J Hum Biol 11, 209-224.

2. Moran A, Jacobs DR, Steinberger J, et al. (1999) Insulin resistance during puberty: results from clamp studies in 357 children. Diabetes 48, 2039-2044.

3. Caprio S, Plewe G, Diamond MP, et al. (1989) Increased insulin secretion in puberty: a compensatory response to reductions in insulin sensitivity. J Pedaitr 114, 963-967.

4. Hoffman RP, Vicini P, Sivitz WI, et al. (2000) Pubertal adolescent male-females differences in insulin sensitivity and glucose effectiveness determined by the one compartment minimal model. Pediatr Res 48, 384-388.

5. Goran MI \& Gower BA (2001) Longitudinal study on pubertal insulin resistance. Diabetes 50, 2444-2450.

6. Hannon TS, Janosky J \& Arslanian SA (2006) Longitudinal study of physiologic insulin resistance and metabolic changes of puberty. Pediatr Res $\mathbf{6 0}, 759-763$.

7. Jeffery AN, Metcalf BS, Hosking J, et al. (2012) Age before stage: insulin resistance rises before the onset of puberty. Diabetes Care 35, 536-541.

8. Ehtisham S, Barrett TG \& Shaw NJ (2000) Type 2 diabetes mellitus in UK children - an emerging problem. Diabet Med 17, 867-871.

9. Kavey REW, Daniels SR, Lauer RM, et al. (2003) American Heart Association guidelines for primary prevention of atherosclerotic cardiovascular disease beginning in childhood. Circulation 107, 1562-1566.

10. Metcalf BS, Hosking J, Henley WE, et al. (2015) Physical activity attenuates the mid-adolescent peak in insulin resistance but by late adolescence the effect is lost: a longitudinal study with annual measures from 9-16 years (EarlyBird 66). Diabetologia 58, 2699-2708.

11. Penno G, Solini A, Bonora E, et al. (2013) Gender differences in cardiovascular disease risk factors, treatments and complications in patients with type 2 diabetes: the RIACE Italian multicentre study. J Intern Med 274, 176-191.

12. Jones M, Barclay AW, Brand-Miller JC, et al. (2016) Dietary glycaemic index and glycaemic load among Australian children and adolescents: results from the 2011-2012 Australian Health Survey. Br J Nutr 116, 178-187.

13. Livesey G, Taylor R, Hulshof T, et al. (2008) Glycemic response and health - a systematic review and meta-analysis: relations between dietary glycemic properties and health outcomes. $\mathrm{Am}$ J Clin Nutr 87, 258S-268S.

14. Amiel SA, Sherwin RS, Simonson DC, et al. (1986) Impaired insulin action in puberty: a contributing factor to poor glycemic control in adolescents with diabetes. $N$ Engl J Med 315, 215-219.

15. Arslanian SA \& Kalhan SC (1994) Correlations between fatty acid and glucose metabolism: potential explanation of insulin resistance of puberty. Diabetes $\mathbf{4 3}, 908-914$.

16. Araujo J, Barros H, Severo M, et al. (2013) Longitudinal changes in adiposity during adolescence: a populationbased cohort. BMJ Open 4, e004380.

17. Moran A, Jacobs DR, Steinberger J, et al. (2002) Association between the insulin resistance of puberty and the insulin-like growth factor-I/growth hormone axis. J Clin Endocrinol Metab 87, 4817-4820.

18. Cooper SB, Bandelow S, Nute ML, et al. (2012) Breakfast glycaemic index and cognitive function in adolescent school children. Br J Nutr 107, 1823-1832.

19. Moore SA, McKay HA, MacDonald H, et al. (2015) Enhancing a somatic maturity prediction model. Med Sci Sports Exerc 47, 1755-1764.

20. Wolever TMS \& Jenkins DJA (1986) The use of the glycaemic index in predicting blood glucose response to mixed meals. Am J Clin Nutr 43, 167-172.

21. International Organisation for Standardisation (ISO) (2010) ISO 26642:2010. Food products - determination of the glycaemic index (GI) and recommendation for food classification. https://www.iso.org/standard/43633.html

22. Foster-Powell K, Holt SHA \& Brand-Miller JC (2002) International table of glycaemic index and glycaemic load values: 2002. Am J Clin Nutr 76, 5-56.

23. Brouns F, Bjorck I, Frayn KN, et al. (2005) Glycaemic index methodology. Nutr Res Rev 18, 145-171.

24. Wolever TMS, Jenkins DJA, Jenkins AL, et al. (1991) The glycemic index: methodology and clinical implications. $\mathrm{Am}$ J Clin Nutr 54, 846-854.

25. Kuwa K, Nakayama T, Hoshino T, et al. (2001) Relationships of glucose concentrations in capillary whole blood, venous whole blood and venous plasma. Clin Chim Acta 307, 187-192.

26. Wolever TMS (2003) Carbohydrate and the regulation of blood glucose and metabolism. Nutr Rev 61, S40-S48.

27. Matthews DR, Hosker JP, Rudenski AS, et al. (1985) Homeostasis model assessment: insulin resistance and beta-cell function from fasting plasma glucose and insulin concentrations in man. Diabetologia 28, 412-419.

28. Stevenson EJ, Williams C, Nute M, et al. (2005) The effect of the glycaemic index of an evening meal on the metabolic responses to a standard high glycaemic index breakfast and subsequent exercise in men. Int J Sport Nutr Exerc Metab 15, 308-322

29. Stevenson EJ, Williams C, Mash LE, et al. (2006) Influence of high-carbohydrate mixed meals with different glycemic indexes on substrate utilization during subsequent exercise in women. Am J Clin Nutr 84, 354-360.

30. Sherar LB, Baxter-Jones ADG \& Mirwald RL (2004) Limitations to the use of secondary sex characteristics for gender comparisons. Ann Hum Biol 31, 586-593.

31. Ruby BC \& Robergs RA (1994) Gender differences in substrate utilisation during exercise. Sports Med 17, 393-410. 